PROCEEDINGS OF THE

AMERICAN MATHEMATICAL SOCIETY

Volume 125, Number 10, October 1997, Pages 2831-2837

S $0002-9939(97) 04269-\mathrm{X}$

\title{
COPRIMENESS AMONG IRREDUCIBLE CHARACTER DEGREES OF FINITE SOLVABLE GROUPS
}

\author{
DIANE BENJAMIN
}

(Communicated by Ronald M. Solomon)

\begin{abstract}
Given a finite solvable group $G$, we say that $G$ has property $P_{k}$ if every set of $k$ distinct irreducible character degrees of $G$ is (setwise) relatively prime. Let $k(G)$ be the smallest positive integer such that $G$ satisfies property $P_{k}$. We derive a bound, which is quadratic in $k(G)$, for the total number of irreducible character degrees of $G$. Three exceptional cases occur; examples are constructed which verify the sharpness of the bound in each of these special cases.
\end{abstract}

\section{INTRODUCTION}

Suppose $G$ is a finite solvable group and let $\operatorname{cd}(G)$ denote the set $\{\chi(1) \mid \chi \in$ $\operatorname{Irr}(G)\}$. We say that $G$ has property $P_{k}$ if every set of $k$ distinct elements of $\operatorname{cd}(G)$ is (setwise) relatively prime. Every finite group $G$ satisfies $P_{k}$ at least for $k \geq|\operatorname{cd}(G)|$, since $1 \in \operatorname{cd}(G)$. The main result of this paper is the following:

Theorem A. Let $G$ be a nonabelian finite solvable group and let $k$ be the smallest positive integer such that $G$ satisfies property $P_{k}$. Then

$$
|\operatorname{cd}(G)| \leq \begin{cases}3 & \text { if } k=2 ; \\ 6 & \text { if } k=3 ; \\ 9 & \text { if } k=4 ; \\ k^{2}-3 k+4 & \text { if } k \geq 5 .\end{cases}
$$

Following the proof of Theorem A, a collection of examples is presented. In each of the exceptional cases $k=2,3,4$ the bound is attained. For $k=2$, an example is provided by the group $S L(2,3)$. This group satisfies $P_{2}$ and has 3 irreducible character degrees: $\operatorname{cd}(S L(2,3))=\{1,2,3\}$. For $k=3$, we construct a group $\Gamma$ with $\operatorname{cd}(\Gamma)=\left\{1, r, s, r s, q^{4}, q^{5}\right\}$, where $q, r, s$ are any three primes satisfying $q \equiv 3(\bmod$ 4) and $q \equiv 1(\bmod r s)$. The group $\Gamma$ attains the bound in this case. Next, for infinitely many values of $k$, we construct a group which satisfies property $P_{k}$ and has $3(k-1)$ irreducible character degrees. Observe that, for $k=4$, such a group satisfies $P_{4}$ and has 9 irreducible character degrees, verifying the sharpness of the bound in this case. It also follows from this infinite set of examples that the best possible bound for $|\operatorname{cd}(G)|$ in terms of $k$ cannot be better than the linear bound $3(k-1)$.

Received by the editors April 4, 1996.

1991 Mathematics Subject Classification. Primary 20C15.

(C)1997 American Mathematical Society 
While this result belongs to a genre of problems and results concerning the irreducible character degrees of finite solvable groups (see $\S 2$ of [4]), it has a unique flavor. The investigation of property $P_{k}$ was inspired by problem 12.3 of [1] which is, in fact, the $k=2$ case of the result. At this point the author would like to express her appreciation to Professor Martin Isaacs for his direction and encouragement in this work, which is a portion of her thesis.

\section{Preliminaries}

The purpose of this section is to restate facts about the structure and character degrees of a factor group $G / K$ of a finite nonabelian solvable group $G$ with $K$ chosen to be maximal such that $G / K$ remains nonabelian. Notice that, in this situation every proper factor group of $G / K$ is abelian and thus $(G / K)^{\prime}$ is the unique minimal normal subgroup of $G / K$.

(2.1) Lemma. Let $G$ be a finite solvable group and assume that $G^{\prime}$ is the unique minimal normal subgroup of $G$. Then all the nonlinear irreducible characters of $G$ have equal degree $f$ and one of the following situations obtains:

(a) $G$ is a p-group, $\mathbf{Z}(G)$ is cyclic and $G / \mathbf{Z}(G)$ is elementary abelian of order $f^{2}$.

(b) $G$ is a Frobenius group with a cyclic Frobenius complement of order $f$. Also, $G^{\prime}$ is the Frobenius kernel and is an elementary abelian p-group.

Proof. This is Lemma 12.3 of [1] with the observation that an abelian Frobenius complement is necessarily cyclic.

(2.2) Theorem. Let $K \triangleleft G$ be such that $G / K$ is a Frobenius group with kernel $N / K$, an elementary abelian p-group. Let $\psi \in \operatorname{Irr}(N)$. Then one of the following holds:

(a) $|G: N| \psi(1) \in \operatorname{cd}(G)$.

(b) $p$ divides $\psi(1)$.

Proof. This is immediate from Theorem 12.4 of [1].

\section{Proof of Theorem A}

We begin by proving a key lemma.

(3.1) Lemma. Let $G$ be a finite nonabelian solvable group with $G^{\prime} \leq \mathbf{O}^{p}(G)$ for all primes $p$. Suppose that $K \triangleleft G$ and $K$ is maximal such that $G / K$ is nonabelian. Then $G / K$ is a Frobenius group with Frobenius kernel $N / K$, an elementary abelian $q$-group for some prime $q$, and a cyclic Frobenius complement. Let $f$ denote the order of the Frobenius complement and assume further that $K$ is chosen so that $f$ is minimal. Then for each linear character $\lambda$ of $N$, either $\lambda^{G}$ is irreducible or $\lambda$ extends to $G$. In particular, if $\chi \in \operatorname{Irr}(G)$ lies over a linear character of $N$, then $\chi$ must have degree 1 or $f$.

It will be handy in the proof to use the standard notation $b(G)$ to denote the largest irreducible character degree of $G$; that is, the maximum of the $\operatorname{set} \operatorname{cd}(G)$.

Proof. By hypothesis, if $M \triangleleft G$ with $K<M$, then the quotient $G / M$ is abelian. Since $G$ is solvable, it follows that $(G / K)^{\prime}$ is the unique minimal normal subgroup of $G / K$. Now since $G$ has no nonabelian $p$-factor groups for any prime $p$, the Frobenius structure of $G / K$ follows from Lemma 2.1 (b). 
Fix a linear character $\lambda \in \operatorname{Irr}(N)$ and let $\chi \in \operatorname{Irr}(G)$ lie over $\lambda$. Set $T=I_{G}(\lambda)$ and $t=|G: T|$. Since $T / N$ is cyclic, $\lambda$ extends to a character $\hat{\lambda} \in \operatorname{Irr}(T)$ and further, by Corollary 6.17 of [1], every element of $\operatorname{Irr}(T \mid \lambda)$ is an extension of $\lambda$. We may then assume without loss of generality that the extension $\hat{\lambda}$ is the Clifford correspondent between $\chi$ and $\lambda$. Thus $\chi=(\hat{\lambda})^{G}$ and $\chi_{N}=\sum_{i=1}^{t} \lambda_{i}$, labeled so that $\lambda_{1}=\lambda$. Also note that $\chi(1)=t$.

We are done if $t=1$ or $t=f$, so assume, for a contradiction, that neither happens. In this case $1<t=\chi(1)<f$ and $N<T<G$. Let $M=\operatorname{ker} \lambda$. Since $T$ fixes the linear character $\lambda$, it follows that $T$ centralizes $N / M$ and so $[T, N] \leq M$. Also $[T, N] \triangleleft G$, since both $T$ and $N$ are normal. Let - denote quotients mod $[T, N]$. Then $\bar{N}$ is central in $\bar{T}$ and $\bar{T} / \bar{N}$ is cyclic since it is isomorphic to $T / N$; thus $\bar{T}$ is abelian. We have $\bar{T}$ is normal and abelian in $\bar{G}$. By Ito's Theorem $(6.15$ of [1]) $t=|\bar{G}: \bar{T}| \geq b(\bar{G})$. Also, since $\operatorname{ker} \chi \geq \operatorname{core}_{G}(\operatorname{ker} \lambda) \geq[T, N]$, we may view $\chi$ as an element of $\operatorname{Irr}(\bar{G})$. We have $t=\chi(1) \in \operatorname{cd}(\bar{G})$ and thus $\bar{G}$ is nonabelian.

Now, let $\bar{G} / \bar{L}$ be a minimal nonabelian factor of $\bar{G}$. Clearly the hypothesis on $p$-factors of $G$ holds for $p$-factors of $\bar{G}$. It follows from Lemma 2.1 that $\bar{G} / \bar{L}$ is a Frobenius group with a Frobenius complement of order $\chi(1)=t<f$. Since factors of $\bar{G}$ are factors of $G$, this contradicts the minimality of $f$.

It will now be convenient to establish some notation for the proof of Theorem A. For a group $G$, we define $k(G)$ to be the smallest integer such that $G$ satisfies property $P_{k}$. Note that $k(G) \leq|\operatorname{cd}(G)|$ and if $G$ is a $q$-group, for $q$ prime, then equality holds. Given a positive integer $q$, we define $\operatorname{cd}_{q}(G)=\{n \in \operatorname{cd}(G) \mid(q, n)=1\}$ and $\operatorname{cd}^{q}(G)=\{n \in \operatorname{cd}(G)|q| n\}$. If $q$ is prime, then $\operatorname{cd}(G)$ is the disjoint union of these two sets. Also, for $N \triangleleft G$ with $m \in \operatorname{cd}(N)$ if there exists $\psi \in \operatorname{Irr}(N)$ and $\chi \in \operatorname{Irr}(G \mid \psi)$ with $\chi(1)=n$ and $\psi(1)=m$, then we will say that $n$ lies over $m$. Further, for each such $m$, define a subset of $\operatorname{cd}(G)$ by $s(m)=\{n \in \operatorname{cd}(G) \mid n$ lies over $m\}$. Note that a given $n$ may lie over many different $m$ and each element of $s(m)$ is divisible by $m$.

To prove Theorem A, we bound each of $\left|\mathrm{cd}^{q}(G)\right|$ and $\left|\operatorname{cd}_{q}(G)\right|$ separately in terms of $k(G)$ and add the results. Note that if $k=k(G)$ for a group $G$, then $\left|\mathrm{cd}^{q}(G)\right| \leq k-1$ for any positive integer $q$. On the other hand, given $k$, examples are available among $q$-groups, where $q$ is prime, which satisfy $|\operatorname{cd}(G)|=k$. For instance, let $Q$ be the direct product of $k-1$ copies of a $q$-group $A$ having $\operatorname{cd}(A)=$ $\{1, q\}$. Then $\operatorname{cd}(Q)=\left\{1, q, q^{2}, \cdots, q^{k-1}\right\}$; thus $k=k(Q)$ and $\left|\operatorname{cd}^{q}(Q)\right|=k-1$. It follows that $k-1$ is the best possible bound for $\left|\operatorname{cd}^{q}(G)\right|$. Our challenge in proving Theorem A will be to bound $\left|\mathrm{cd}_{q}(G)\right|$.

Proof of Theorem A. Let $G$ be a nonabelian finite solvable group and let $k=k(G)$.

Suppose first that $G$ has a nonabelian $p$-factor group $G / K$ for some prime $p$. As we have observed, $\left|\operatorname{cd}^{p}(G)\right| \leq k-1$. Now we consider $\left|\operatorname{cd}_{p}(G)\right|$. Fix $\psi \in \operatorname{Irr}(G / K)$ with $\psi(1)=p^{a}>1$. For each character $\chi \in \operatorname{Irr}(G)$ with $(p, \chi(1))=1$ we have $\chi_{K} \in \operatorname{Irr}(K)$. By Corollary 6.17 of $[1]$, we have $\chi \psi \in \operatorname{Irr}(G)$ with degree $\chi \psi(1)$ divisible by $p$, since $\chi \psi(1)=\chi(1) p^{a}$. This gives an injection from $\operatorname{cd}_{p}(G)$ into $\operatorname{cd}^{p}(G)$. Thus $\left|\operatorname{cd}_{p}(G)\right| \leq k-1$ and $|\operatorname{cd}(G)| \leq 2(k-1)$. In this case the conclusion of the theorem holds. Henceforth we assume that $G^{\prime} \leq \mathbf{O}^{p}(G)$ for all primes $p$.

Now fix $K \triangleleft G$ so that $K$ is maximal with $G / K$ nonabelian. By Lemma 2.1, $G / K$ is a Frobenius group with kernel $N / K$, an elementary abelian $q$-group, and with a 
cyclic complement $H / K$ of order $f$. Also $\operatorname{cd}(G / K)=\{1, f\}$. Assume further that $K$ is chosen so that $f$ is minimal. As before, we have $\left|\mathrm{cd}^{q}(G)\right| \leq k-1$.

To assess $\left|\operatorname{cd}_{q}(G)\right|$ we will examine how many distinct elements of $\operatorname{cd}_{q}(G)$ lie over each element of $\operatorname{cd}(N)$. If we write $\operatorname{cd}(N)=\operatorname{cd}^{q}(N) \dot{\cup} \operatorname{cd}_{q}(N)$, then notice that elements of $\operatorname{cd}^{q}(G)$ can lie over only elements of $\operatorname{cd}^{q}(N)$, since $(q, f)=1$, and elements of $\operatorname{cd}_{q}(G)$ lie over only elements of $\operatorname{cd}_{q}(N)$. Also, by Theorem 2.2 (a), for each element $z \in \operatorname{cd}_{q}(N)$ we must have $f z \in \operatorname{cd}(G)$. This gives an injection from $\operatorname{cd}_{q}(N)$ into $\operatorname{cd}^{f}(G)$. Again, by hypothesis, $\left|\operatorname{cd}^{f}(G)\right| \leq k-1$; thus $\left|\operatorname{cd}_{q}(N)\right| \leq k-1$. It follows that all the elements of $\operatorname{cd}_{q}(G)$ lie over the, at most $k-1$, elements of $\operatorname{cd}_{q}(N)$.

If $z \in \operatorname{cd}_{q}(N)$, how many elements of $\operatorname{cd}_{q}(G)$ can lie over $z$ ? By Lemma 3.1, if $z=1$, then $s(z)=\{1, f\}$. If $z>1$, then $|s(z)| \leq k-1$, since $s(z) \subseteq \operatorname{cd}^{z}(G) \leq k-1$, by hypothesis. It follows that $\left|\operatorname{cd}_{q}(G)\right| \leq 2+(k-2)(k-1)$ and thus we have:

$$
\text { (*) }|\operatorname{cd}(G)| \leq\left|\operatorname{cd}^{q}(G)\right|+\left|\operatorname{cd}_{q}(G)\right| \leq(k-1)+2+(k-2)(k-1)=k^{2}-2 k+3 .
$$

Observe that, when $k=2$ the bound $(*)$ yields $|\operatorname{cd}(G)| \leq 3$ and when $k=3$ the bound $(*)$ yields $|\operatorname{cd}(G)| \leq 6$. Thus the first two special cases of Theorem A have been proved. Henceforth we assume that $k \geq 4$ and will improve $(*)$. We continue as before with the Frobenius factor group $G / K$.

If $\left|\mathrm{cd}_{q}(N)\right|<k-1$, then each of the, at most $k-3$, nonlinear character degrees of $\operatorname{cd}_{q}(N)$ has at most $k-1$ elements of $\operatorname{cd}_{q}(G)$ lying over it; thus $\left|\operatorname{cd}_{q}(G)\right| \leq$ $2+(k-3)(k-1)$. This observation along with our bound on $\operatorname{cd}^{q}(G)$ yields $|\operatorname{cd}(G)| \leq$ $(k-1)+2+(k-3)(k-1)=k^{2}-3 k+4$ and there is nothing further to prove in this case.

We may now assume that $\left|\operatorname{cd}_{q}(N)\right|=k-1$. In this case $\left\{f x \mid x \in \operatorname{cd}_{q}(N)\right\}$ is a subset of $\operatorname{cd}(G)$ of size $k-1$. We will show that $s(z) \subseteq\{z\} \cup\left\{f x \mid x \in \operatorname{cd}_{q}(N)\right\}$ for each $z \in \operatorname{cd}_{q}(N)$. Recall that an arbitrary member of $s(z)$ has the form $r z$, where $r \mid f$. If $r z \in s(z)$ with $r>1$, then $r$ divides every member of $\{r z\} \cup\left\{f x \mid x \in \operatorname{cd}_{q}(N)\right\}$. Since the latter set in this union has size $k-1$, it follows that $r z \in\left\{f x \mid x \in \operatorname{cd}_{q}(N)\right\}$ and thus we conclude that $s(z) \subseteq\{z\} \cup\left\{f x \mid x \in \operatorname{cd}_{q}(N)\right\}$ as claimed. It follows that all the members of $\operatorname{cd}_{q}(G)$ lie in $\operatorname{cd}_{q}(N) \cup\left\{f x \mid x \in \operatorname{cd}_{q}(N)\right\}$; hence $\left|\operatorname{cd}_{q}(G)\right| \leq$ $2(k-1)$. Since $\left|\operatorname{cd}^{q}(G)\right| \leq k-1$, we have $|\operatorname{cd}(G)| \leq 3 k-3$, in this case.

For $k \geq 4$ (and $\mathbf{O}^{p}(G)=1$ ), it follows that $|\operatorname{cd}(G)|$ is bounded by the maximum of the bounds derived in the two preceding paragraphs. That is,

$$
|\operatorname{cd}(G)| \leq \max \left\{\begin{array}{l}
(k-1)+2+(k-3)(k-1)=k^{2}-3 k+4, \\
(k-1)+2(k-1)=3 k-3 .
\end{array}\right.
$$

Observe that, for $k=4$, the second formula yields a maximum of 9 , giving $|\operatorname{cd}(G)| \leq$ 9. In the cases $k \geq 5$, the maximum is $k^{2}-3 k+4$. Thus Theorem $\mathrm{A}$ is proved.

In the next section we give constructions which verify the sharpness of the bound in the exceptional cases $k=3$ and $k=4$.

\section{Constructions}

For any three primes $q, r, s$ satisfying $q \equiv 3(\bmod 4)$ and $q \equiv 1(\bmod r s)$, we construct a group $\Gamma$ as the semidirect product of a normal Sylow $q$-subgroup $Q$ and a cyclic group $H$ of order $r s$ such that $\operatorname{cd}(\Gamma)=\left\{1, r, s, r s, q^{4}, q^{5}\right\}$. The group $\Gamma$ satisfies $P_{3}$ and has 6 irreducible character degrees; thus providing an example for 
the sharpness of the bound in the case $k=3$. Note that $r=2, s=3, q=7$ satisfy the conditions.

First we construct $Q$. Let $q$ be prime with $q \equiv 3(\bmod 4)$. Define the group $Q$ of exponent $q$ as follows, where all unspecified commutators are trivial:

$$
\begin{gathered}
Q=\left\langle x_{1}, x_{2}, x_{3}, x_{4}, x_{5}, x_{6}, x_{7}, x_{8}, x_{9}, x_{10}\right| \\
{\left[x_{1}, x_{2}\right]=\left[x_{3}, x_{4}\right]=\left[x_{5}, x_{6}\right]=\left[x_{7}, x_{8}\right]=\left[x_{9}, x_{10}\right],} \\
\left.\left[x_{1}, x_{4}\right]=\left[x_{2}, x_{3}\right]=\left[x_{5}, x_{8}\right]=\left[x_{6}, x_{7}\right]\right\rangle .
\end{gathered}
$$

A few observations about the group $Q$ are helpful. First, for notational convenience, label $z_{1}=\left[x_{1}, x_{2}\right]=\left[x_{3}, x_{4}\right]=\left[x_{5}, x_{6}\right]=\left[x_{7}, x_{8}\right]=\left[x_{9}, x_{10}\right]$ and $z_{2}=\left[x_{1}, x_{4}\right]=$ $\left[x_{2}, x_{3}\right]=\left[x_{5}, x_{8}\right]=\left[x_{6}, x_{7}\right]$. Notice that $\mathbf{Z}(Q)=Q^{\prime}=\left\langle z_{1}, z_{2}\right\rangle$. From this we see that $|\mathbf{Z}(Q)|=q^{2}$ and $|Q / \mathbf{Z}(Q)|=q^{10}$. Thus the group $Q$ is $q$-special of order $q^{12}$ with exponent $q$. Further, using additive notation, we may view $Q / \mathbf{Z}(Q)$ and $\mathbf{Z}(Q)$ as vector spaces over $G F(q)$ with bases $\left\{\overline{x_{1}}, \overline{x_{2}}, \cdots, \overline{x_{10}}\right\}$ and $\left\{z_{1}, z_{2}\right\}$, respectively. (Here ${ }^{-}$denotes quotient $\bmod \mathbf{Z}(Q)$.)

What are the degrees of the irreducible characters of $Q$ ? Since $Q$ is $q$-special with $|Q: \mathbf{Z}(Q)|=q^{10}$ it follows that $Q$ has $q^{10}$ linear characters. Notice that $Q /\left\langle z_{1}\right\rangle$ is isomorphic to the direct product of an extra-special group of order $q^{9}$ having exponent $q$ with $Z_{q} \times Z_{q}$. Also $Q /\left\langle z_{2}\right\rangle$ is an extra-special group of order $q^{11}$ having exponent $q$. These quotients give some information about $\operatorname{Irr}(Q)$ and about $\operatorname{cd}(Q)$. In particular, we have $\left\{1, q^{4}, q^{5}\right\} \subseteq \operatorname{cd}(Q)$. In fact, with the assumption $q \equiv 3 \bmod 4$, we can show that these are the only irreducible character degrees of $Q$. The following fact is required:

Claim. For each nonlinear character $\theta \in \operatorname{Irr}(Q)$ we have

(i) $Q / \operatorname{ker}(\theta)$ is an extra-special $q$-group with center $\mathbf{Z}(\theta) / \operatorname{ker}(\theta)$.

(ii) $\mathbf{Z}(\theta) \leq\left\langle z_{1}, z_{2}, x_{9}, x_{10}\right\rangle$.

In particular, we have $\theta(1)$ is $q^{4}$ or $q^{5}$ and every automorphism that centralizes $\left\langle z_{1}, z_{2}, x_{9}, x_{10}\right\rangle$ fixes $\theta$.

Fix a nonlinear character $\theta \in \operatorname{Irr}(Q)$. Since $Q^{\prime}=\mathbf{Z}(Q)$ we have $\mathbf{Z}(Q) \not \leq \operatorname{ker}(\theta)$; also $\mathbf{Z}(Q) \cdot \operatorname{ker}(\theta) \leq \mathbf{Z}(\theta)$. The nontrivial group $\mathbf{Z}(\theta) / \operatorname{ker}(\theta)$ is cyclic of exponent $q$; thus it has order $q$. Further $\mathbf{Z}(\theta)=\operatorname{ker}(\theta) \cdot \mathbf{Z}(Q)$. It follows that $Q / \operatorname{ker}(\theta)$ has a center of order $q$ and that the factor group modulo the center is elementary $q$-abelian. Thus part (i) holds and from this we have $\theta(1)^{2}=|Q: \mathbf{Z}(\theta)|$. Now since $\left|\left\langle z_{1}, z_{2}, x_{9}, x_{10}\right\rangle\right|=q^{4}$, the final statement of the claim will hold once part (ii) is established.

As before, let ${ }^{-}$denote quotient $\bmod \mathbf{Z}(Q)$. Using additive notation in each of the abelian groups $\bar{Q}$ and $\mathbf{Z}(Q)$, for an element $y \in \mathbf{Z}(\theta)$ we may write:

$$
\bar{y}=c_{1} \overline{x_{1}}+c_{2} \overline{x_{2}}+\cdots+c_{10} \overline{x_{10}} \quad \text { with } \quad c_{i} \in G F(q) .
$$

For each element $z \in \mathbf{Z}(Q)$ and for each generator $x_{i}$ we have $\left[x_{i}, y z\right]=\left[x_{i}, y\right]$. Using the defining relations for the group $Q$, it follows that:

$$
\left[x_{1}, y\right]=c_{2} z_{1}+c_{4} z_{2} \quad \text { and } \quad\left[x_{3}, y\right]=c_{4} z_{1}-c_{2} z_{2} .
$$

Observe that for each element $y \in \mathbf{Z}(\theta)$ and $x \in Q$ we have $[y, x] \in Q^{\prime} \cap \operatorname{ker}(\theta)=$ $\mathbf{Z}(Q) \cap \operatorname{ker}(\theta)$; thus each of $\left[x_{1}, y\right]$ and $\left[x_{3}, y\right]$ lie in $\mathbf{Z}(Q) \cap K$. Viewing $\mathbf{Z}(Q) \cap K$ as a 1-dimensional subspace of $\mathbf{Z}(Q)$, the vectors $c_{2} z_{1}+c_{4} z_{2}$ and $c_{4} z_{1}-c_{2} z_{2}$ are dependent. If either $c_{2}$ or $c_{4}$ is nonzero, then both are nonzero and $c_{4}=\alpha c_{2}$ and $c_{2}=-\alpha c_{4}$ for some $\alpha \in G F(q)$; in which case $-\alpha^{2}=1$. Invoking the hypothesis 
$q \equiv 3 \bmod 4$, there is no solution for $-\alpha^{2}=1$ in $G F(q)$. This forces $c_{2}=c_{4}=0$. Under the same assumption, similar comparisons (e.g. $\left[x_{2}, y\right]$ and $\left[x_{4}, y\right]$ ) force $c_{1}=c_{3}=c_{5}=c_{6}=c_{7}=c_{8}=0$. Thus we have $\mathbf{Z}(\theta) \leq\left\langle x_{9}, x_{10}, z_{1}, z_{2}\right\rangle$ and the claim is proved.

Now let $r$ and $s$ be distinct primes dividing $q-1$. We will define the action of a cyclic group $H$ of order $r s$ on the group $Q$. First, we define actions on $Q$ by automorphisms $a$ and $b$ of orders $r$ and $s$ respectively. Let $\delta$ and $\epsilon$ be primitive $r$ and $s$ roots of unity in $G F(q)$ respectively. The actions of $a$ and $b$ are defined on the generators of $Q$, where all unspecified generators are fixed:

$$
\begin{aligned}
& \text { for } a: x_{1}^{a}=x_{1}^{\delta}, x_{2}^{a}=x_{2}^{\delta^{-1}}, x_{3}^{a}=x_{3}^{\delta}, x_{4}^{a}=x_{4}^{\delta^{-1}} ; \\
& \text { for } b: x_{5}^{b}=x_{5}^{\epsilon}, x_{6}^{b}=x_{6}^{\epsilon^{-1}}, x_{7}^{b}=x_{7}^{\epsilon}, x_{8}^{b}=x_{8}^{\epsilon^{-1}} .
\end{aligned}
$$

We must verify that the proposed definitions interact well with the defining relations among the generators. That is, it must be shown that: if $\left[x_{i}, x_{j}\right]=\left[x_{k}, x_{l}\right]$, then $\left[x_{i}, x_{j}\right]^{a}=\left[x_{k}, x_{l}\right]^{a}$ and $\left[x_{i}, x_{j}\right]^{b}=\left[x_{k}, x_{l}\right]^{b}$. In fact, more is true. If we write $x_{i}^{a}=x_{i}^{\alpha_{i}}$ and $x_{j}^{a}=x_{j}^{\alpha_{j}}$, then $\left[x_{i}, x_{j}\right]^{a}=\left[x_{i}^{a}, x_{j}^{a}\right]=\left[x_{i}^{\alpha_{i}}, x_{j}^{\alpha_{j}}\right]=\left[x_{i}, x_{j}\right]^{\alpha_{i} \alpha_{j}}$; and whenever $\left[x_{i}, x_{j}\right] \neq 1$ we have $\alpha_{i} \alpha_{j}=1$. Thus every commutator $\left[x_{i}, x_{j}\right]$ is fixed by $a$. The same holds for $b$. It follows that $a$ and of $b$ act as automorphisms on $Q$ and that the subgroup $\left\langle z_{1}, z_{2}, x_{9}, x_{10}\right\rangle$ is centralized by both $a$ and $b$. It is also clear that $a b=b a$. Since $a$ and $b$ are commuting automorphisms of $Q$ of relatively prime orders, the cyclic group $H=\langle a\rangle \times\langle b\rangle$ acts on $Q$. Note that $|H|=r s$ and $H$ centralizes $\left\langle z_{1}, z_{2}, x_{9}, x_{10}\right\rangle$.

Now we consider the action of $H$ on $\mathbf{Z}(Q)$ and on $Q / \mathbf{Z}(Q)$. As observed, $H$ centralizes $\mathbf{Z}(Q)$. To understand the action of $H$ on $Q / \mathbf{Z}(Q)$, we return to a vector space point of view. As before, let ${ }^{-}$denote quotients $\bmod \mathbf{Z}(Q)$ and use additive notation. From this perspective, the set $\left\{\overline{x_{1}}, \overline{x_{2}}, \ldots, \overline{x_{10}}\right\}$ is a basis for $Q / \mathbf{Z}(Q)$ consisting of eigenvectors of $a$ and $b$ with corresponding eigenvalues-

$$
\begin{aligned}
& \text { for } a: \delta, \delta^{-1}, \delta, \delta^{-1}, 1,1,1,1,1,1 \text {; } \\
& \text { for } b: 1,1,1,1, \epsilon, \epsilon^{-1}, \epsilon, \epsilon^{-1}, 1,1 .
\end{aligned}
$$

It follows that $a$ and $b$ act diagonally on $\bar{Q}$ and that the orbits of the action of $H$ on $\bar{Q}$ have sizes $1, r, s$, and $r s$.

To complete the construction, define the group: $\Gamma=Q \rtimes H$.

Now we consider $\operatorname{cd}(\Gamma)$. The coprime action of $H$ on the abelian group $Q / \mathbf{Z}(Q)$ is permutation isomorphic to the action of $H$ on $\operatorname{Irr}(Q / \mathbf{Z}(Q))$. The orbit sizes of the former action are $\{1, r, s, r s\}$. Since $H$ is cyclic, it follows that $\operatorname{cd}(\Gamma / \mathbf{Z}(Q))=$ $\{1, r, s, r s\}$. On the other hand, we see from the claim that each nonlinear irreducible character of $Q$ is fixed by $H$, since $H$ centralizes $\left\langle z_{1}, z_{2}, x_{9}, x_{10}\right\rangle$. Again, since $H$ is cyclic and since the action of $H$ on $Q$ is coprime, the remaining elements of $\operatorname{cd}(\Gamma)$ are exactly the nonlinear irreducible character degrees of $\operatorname{cd}(Q)$. It follows that $\operatorname{cd}(\Gamma)=\left\{1, r, s, r s, q^{4}, q^{5}\right\}$.

The next construction will show that for infinitely many values of $k$ there exist groups which satisfy property $P_{k}$ and have $3(k-1)$ irreducible character degrees. It follows that the bound of Theorem A cannot be better than the linear bound $3(k-1)$.

For any two distinct primes $p$ and $q$ and for an appropriate $n$, one can let a cyclic group of order $p$ act on an extra-special $q$-group of order $q^{(2 n+1)}$ such that the 
irreducible character degrees of the resulting semidirect product are $\left\{1, p, q^{n}\right\}$. Let the group $G$ be the direct product of $m$ groups of this sort such that all of the primes involved are distinct. Then $|\operatorname{cd}(G)|=3^{m}$; further, since a single prime divides no more than $3^{(m-1)}$ irreducible character degrees of $G$, we have $k=k(G)=3^{(m-1)}+1$. It follows that $|\operatorname{cd}(G)|=3(k-1)$, showing that the bound of Theorem A can be no better than $3(k-1)$.

Finally, observe that if, as in the preceding construction, we let $\Delta=A \times B$ for groups $A$ and $B$ with $\operatorname{cd}(A)=\{1,2,3\}$ and $\operatorname{cd}(B)=\{1,5,11\}$, then $\Delta$ satisfies $P_{4}$ and has $|\operatorname{cd}(\Delta)|=9$. Thus $\Delta$ verifies the sharpness of the bound in the case $k=4$ and completes our collection of examples for each of the exceptional cases of Theorem A.

\section{REFERENCES}

[1] I. M. Isaacs, "Character Theory of Finite Groups," Academic Press, New York, 1976. MR $\mathbf{5 7 : 4 1 7}$

[2] B. Huppert, "Endliche Gruppen I," Springer-Verlag, Berlin-Heidelberg-New York-Tokyo, 1967. MR 37:302

[3] I. M. Isaacs and D. S. Passman, "A characterization of groups in terms of the degrees of their characters II," Pacific J. of Math. 24, No.3, (1968) 467-510. MR 39:2864

[4] B. Huppert, "Research in Representation Theory at Mainz (1984 - 1990)," Progress in Mathematics. 95, (1991) 17-36. MR 92c:20011

Department of Mathematics, University of Wisconsin-Platteville, Platteville, WisCONSIN 53818

E-mail address: benjamin@uwplatt.edu 